\title{
EFFECT OF DIFFERENT SPOTTING HEIGHTS ON BALLET PIROUETTE PERFORMANCE
}

\author{
Antonio Cicchella, Cioia Caminiti \\ Department for Quality of Life Studies, University of Bologna, Italy
}

\begin{abstract}
Having a focus of attention (FOA) in executing complex motor tasks can facilitate task execution. This behaviour influences subsequent movements. We studied the effect of different fixation points on the posture of ten experienced female dancers during the performance of a pirouette. Specifically, the shifting of forces between supporting and gesture legs during the preparation phase and the times of execution of the pirouette were studied. The preparation phase showed a closer placement of the feet than found in previous studies (28\% vs $40 \%$ of the leg length) and a preferred spotting height of $55 \%$ of the body height. Pirouettes with a lowspotting height showed a longer start and times to peak force of the supporting leg. No statistical differences were found for bending time on the pirouettes with different spotting heights. The hell landing forces for the gesture leg were different $(\mathrm{p}<0.01)$ between trials for the four different spotting heights. Finally, small adjustments in external FOA $(<30 \mathrm{~cm}$ at $3.5 \mathrm{~m}$ of distance) did not influence the performance of the pirouettes significantly but greater gaps did.
\end{abstract}

Keywords: dance; biomechanics; pirouettes; focus of attention

\section{INTRODUCTION}

One of the most common turning movements in all forms of dance is a pirouette, a rotation of the body around a vertical axis over one supporting foot on the floor. A theoretical model for turns has been clearly described: "posture anticipates gesture, interacting systems allow both preset and changing strategies, peripheral inputs allow immediate corrections, the final position is programmed in advance, and differing areas of the brain are involved in 
the execution of turns" [9]. Dance female students represent a large population whose performance on the motor task of a single pirouette is highly generalizable to the majority of the dancer female population. Ballet dancers train for many years to be able to do a pirouette rapidly and repeatedly, and the absence of vertigo is critical for maintaining the balance and spatial orientation required by ballet training. This is achieved by using a technique called spotting, which minimizes head movements.

Advantages of adopting external focus vs an internal focus of attention (FOA) induced by instructions or feedback, have been studied for a variety of motor skills, skill levels and populations [14]. However, there have been few investigations on the FOA involved in the performing arts. The only existing study, concentrate on comparing the internal FOA on different body parts, concluding that a generalized attention, instead of focus on a specific body part, is most effective for learning the pirouette [1]. In ballet, from the late 19th century, when classical dance began to emphasize virtuosity, it has been generally recognized that an external FOA helps dancers during their pirouettes. This technique is effective because it requires dancers to snap their head and eyes around, faster than the rest of their body, to face front again. A recent study [11], investigating brain structures, demonstrated that this spotting technique is not only an aesthetic necessity, but also helps ballet dancers avoid dizziness when they perform the pirouettes.

An early study by McMillan [8] used 3D cinematography to compare the execution of the pirouette in three skill levels of dancers during the preparation, turn, and conclusion phases of the turn. Participants were three professional dancers, three semi-skilled university dance majors, and three beginners. They performed three pirouettes from fourth position in each of the views. Kinematics showed that the preparation phase was more similar for the three groups than the turn and conclusion phases. However, professional dancers demonstrated larger movements and took less time in the preparation phase than semi-skilled and beginner dancers. The skilled dancers were different from the dance majors and beginners in the spotting technique, arm position, right foot placement, and acceleration during the turn. The dance majors and beginners were not different from each other for these measures. Laws [4] studied the torque produced and the resulting angular momentum in a female professional ballet dancer performing the pirouette en de hors, arabesque turn, and pirouette en dedans with gesture leg in low second position. Fifty trials were completed over two days. Laws [4] described the turns qualitatively, and made suggestions about how to perform these turns with correct technique and efficiency. Laws [5] examined 
the mechanics of the fouetté turn, collecting data with videography from the front and directly overhead.

The literature contains many investigations into the difference in the muscle strength and power of the dominant and non-dominant sides, when performing a variety of sporting and daily movements $[2,7]$. Pirouettes are an important movement in ballet affected by "lateral bias" and a difference exists in the muscle effort and coordination ability required of the supporting leg (the leg working in open chain) and gesture leg (the leg controlling posture in closed-chain), when performing difficult asymmetric movements such as turns [3, 7]. Spotting height can significantly affect the weight distribution between supporting and gesture legs in the pirouette. The foot displacement of the experienced group with dominant support was found to be significantly smaller than that with non-dominant leg support. In contrast, no significant difference was observed in the foot displacement of the novice group given dominant leg support or non-dominant leg support [6]. Findings of Lin et al. [6] show that in performing pirouettes with endehors, a significant lateral bias exists only in experienced dancers. Lateral bias in handwriting, kicking, hopping and throwing is already developed before school age [12].

However, training further reinforces this innate lateral bias. This finding suggests the potential presence of an effect from training and rehearsal in the dancers. Farrar-Baker and Wilmerding [2] counted the number of repetitions to the right and to the left in each combination instructed by the teacher in a ballet training class. They found that the number of repetitions performed to the right was $26 \%$ higher than that of the repetitions performed to the left. The great tendency of ballet dancers to use the right side is indeed correlated with the instructional methods used in classical ballet training, from the barré to the centre. Repetitive rehearsal on the side of preference strengthens the side dominance in experienced dancers, probably because they have a greater opportunity to perform solo dance than novice dancers, who are generally required to dance in corps de ballet.

Lin et al. [6] looked at the different postural control strategies exhibited by 13 experienced female ballet dancers and 13 novice female ballet dancers. They used a data acquisition system with eight high-speed cameras and 36 markers on anatomical landmarks and two force plates to record the ground reaction force. All participants performed a single-revolution pirouette endehor. Five successful trials were performed by each dancer. The turns were performed on the force plates. In agreement with a previous study [5], professional dancers took less time in the preparation phase of the pirouette 
endehors, while novice dancers had a longer preparatory phase. This may suggest that novice dancers need more time to adjust their posture before turning. They might also spend more time in the preparation phase for planning their movements. The novice dancers applied a greater push force than experienced dancers. The smaller anterior/posterior ground reaction force needed for the turning by the experienced dancers demonstrated that the experienced dancers exerted smaller peak forces in the anterior-posterior direction with both legs. By contrast, the greater anterior-posterior forces in the novice dancers indicate that they put more effort into maintaining their postures and dynamic balance.

There are no studies that have investigated the spotting or the visual strategies used in performing the pirouette. The purpose of this study was to investigate the influence of the height of external FOA on the statics, kinematics and dynamics of the pirouette. Specifically, we analyzed the preparation stance and landing differences with reference to the ground reaction forces, the centre of pressure path on front and back legs. Differences in times in the five different phases of the pirouettes were also studied.

\section{MATERIALS AND METHODS}

\section{Subjects}

Ten experienced female dancers took part in the study (Table 1). The inclusion criteria were specified as follows: 1 ) a classical ballet training history of at least six years; 2) a routine participation in ballet training for a minimum of three hours per week; and 3), the ability to perform at least two complete revolutions of turning with single leg support (pirouettes). The exclusion criteria of the participant were a history of a vestibular or balance problems and previous injuries of the lower extremity and back, which would affect their performance in executing the assigned tasks. Prior to participation some basic anthropometric data were measured with an anthropometer (Martin Metal) and a medical scale (Seca): body height and weight, leg length (from anterior superior iliac spine to the sole of the foot) and visual axis height (height from the ground of the cross point between the Frankfurt Plane and mid-sagittal plane) (Table 1). All dancers (100\%) passed with "A" as their final marks in their dance class. The subjects went to the laboratory for testing in the morning. They performed the tests starting looking at their preferred spotting height, then moved to the higher spotting height, to the lower, and in the end to the lowest FOA. Each trial lasted three seconds. It took $40 \mathrm{~min}$ for each subject to complete all the trials. 
Table 1. Subjects and posture measurements. Means and SD

\begin{tabular}{lc}
\hline Age $(\mathrm{yrs})$ & $19.2 \pm 1.1$ \\
\hline Height $(\mathrm{cm})$ & $161.98 \pm 7.56$ \\
\hline Mass $(\mathrm{kg})$ & $56.02 \pm 4.04$ \\
\hline BMI $\left(\mathrm{kg} / \mathrm{m}^{2}\right)$ & $21.39 \pm 1.45$ \\
\hline Visual axis height $(\mathrm{cm})$ & $148.3 \pm 8.81$ \\
\hline Visual axis from the 4th position (cm) & $158.3 \pm 8.81$ \\
\hline Preferred spot height (cm) & $87.10 \pm 11.84$ \\
\hline Preferred height at start (\% of visual axis) & $55 \pm 10$ \\
\hline Feet Preparation width in 4th pos. (cm) & $26.3 \pm 7.6$ \\
\hline Feet Preparation width (\% of leg length) & $28 \pm 7$ \\
\hline Leg Length (cm) & $94 \pm 4.78$ \\
\hline
\end{tabular}

\section{Procedures}

Each participant read and signed an informed consent approved by the Institutional Review Board. Subjects performed a single revolution pirouette endehors by spinning the gesture leg outward and backward, which is something they usually do everyday trainings during their dance classes. During the execution of each task, the 3D trajectories of the markers were captured using an acquisition a Qualisys Motion Capture System (Sweden) with eight Oqus cameras with a sampling rate of $100 \mathrm{~Hz} .18$ markers were attached to the skin, leotard or ballet shoes on the following relevant anatomical landmarks: umbelicus, sacrum, anterior superior iliac spine, midpoints of the lateral sides of the thigh and shank, grand throchanter, lateral knee, lateral malleoli, posterior hell, and 1st and 5th metatarsal heads midpoints. The same researcher placed all the markers for all participants in order to increase the reliability. After prospering warming up and after two static standing trials, the dancer performed a full rotation of the body at their preferred speed with single-leg support on the toes of the foot and landing on two force plates, $1000 \mathrm{~Hz}$ sampling rate. The force was calculated using Qualisys software (Qualisys, Sweden). All female participants performed a single-revolution pirouette endehors by spinning the gesture leg outward and backward with the support of the dominant leg. The participant's dominant leg was identified as the leg chosen for kicking an object without a second thought [7]. 
Selected dancers preferred turning in clockwise direction and so they were tested with the right-leg as the dominant leg and the left-leg as a support leg in this study. Subjects were requested to stand on two force plates in a comfortable 4 th ballet position and the distances between the heel of the front foot and the toe of the back foot is measured and recorded placing a black taping on the ground. Subjects indicate on the board in front of them $(3.5 \mathrm{~m}$ ahead) their preferred spot height. The researcher recorded the point with a fluorescent dot $(2 \mathrm{~cm})$. Dancers performed 20 trials of pirouettes changing the height of their visual spot, starting from their preferred height of spotting. Five successful trials of pirouette endehors were acquired and averaged in each dancer at the preferred spotting height, $30 \mathrm{~cm}$ above, 30 and $60 \mathrm{~cm}$ below. A successful trial was considered when the dancer performed the pirouette on the two force plates and land on them as well. Start the pirouette endehors, using the spot sign on the board, upon hearing a verbal "ready" command from the experimenter, finish the pirouette endehors in a 4 th ballet position with straight leg. 4th position feet width, vertical force component, temporal parameters and feet displacements were recorded continuously as participants performed a single pirouette endehors.

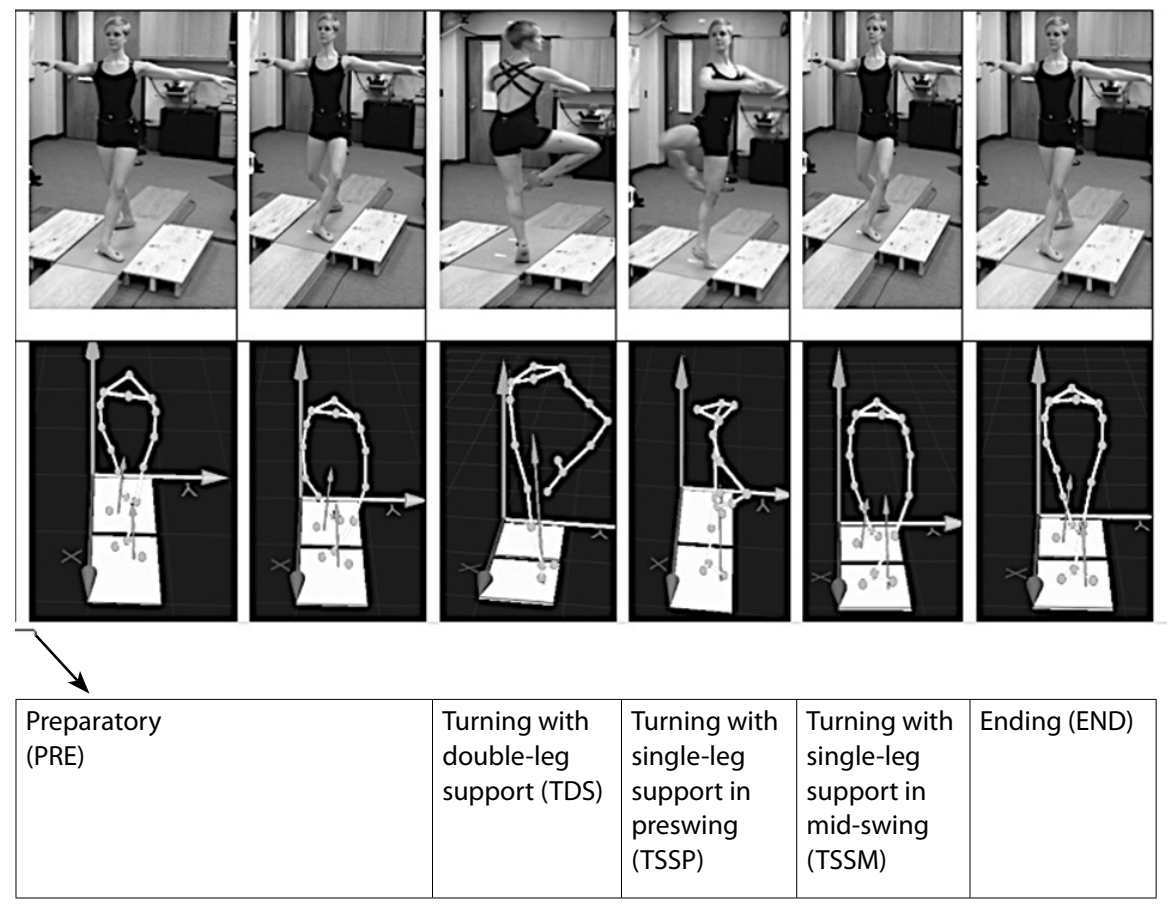

Figure 1. Phases of pirouette 
The pirouette was divided into five phases, according to Lin [7] (Figure 1): the preparatory phase (PRE), the turning with double-leg support (TDS), the turning with single-leg support in pre-swing phase (TSSP), the turning with single-leg support in mid-swing (TSSM), and the ending phase (END). Centre of pressure (COP) of the body on the $\mathrm{x}$ and $\mathrm{y}$ axis (anteroposterior and mediolateral), vertical forces and times were computed at the start and at the landing and for the 5 phases of the pirouettes.

The times of the main phases of the pirouette endehors were calculated (Table 3): bending time (BT) is the time that start from when the dancers listen an audible cue to the point of maximum bending of their knees. It corresponds with the peak of the gesture leg during the "turning with double leg support phase". Bending time is a significant parameter when comparing novice and experienced dancers. Start time (ST) was defined as the time in which dancers' back toe is leaving the ground to go into retire position, right after the gesture leg's peak when all gesture leg's forces are zero. Supporting leg peak time (SPT) is the time in which all dancers' body weight is on their supporting leg. Toe and heel landing (TLT, HLT) are referred respectively to the land of the toe and heel of the gesture leg, when it retouches ground after the pirouette at the beginning of the ending phase. Recovery time (RT) indicates the time, after the turn, to straight again the knees. Professional dancers take less time in the preparation phase than do less skilled dancers because "they need more time to adjust their posture before turning" [13] and for cognitive thinking because "they were in early stage of their acquisition" [10].

\section{Statistical analysis}

A repeated measure ANOVA with significance level of 0.05 using Bonferroni correction was performed for the COP displacements forces and times variables with the SPSS 20 software.

\section{RESULTS}

We compared the preferred spotting height with dancers' visual axis height (Table 1). When dancers search a spotting point for their pirouettes they look at a point in line with their visual axis height because they start every single pirouette with a plié position. So when they bend their knees they actually focus at a spotting point that is $55 \pm 10 \%$ of their visual axis height. Before starting the trials, they stand on two forces plates that are $10 \mathrm{~cm}$ above the ground, so their visual axis height in ballet 4 th position is the 
visual axis height plus $10 \mathrm{~cm}$ (Table 1). In the preparation position, our subjects preferred a mean distance between the feet of $28 \pm 7 \%$ of a dancer's leg length. Therefore, $10 \%$ of the subjects started with a $31 \%$ and another $40 \%$ started with a width that was $26-27 \%$ of their leg length. Previous studies [5] suggested that dancers complete a better successful pirouette endehors when they started with a width of their 4th position that corresponds to the $40 \%$ of their leg length or to the $35 \%$ of their leg length. Only $20 \%$ of our dancers started their pirouettes in accordance with the recommended measures. Significant differences were found only in the $\mathrm{x}$ displacements (anteriorposterior) of COP between all spotting conditions.

The vertical component of the force was calculated for the supporting (S) and gesture $(\mathrm{G})$ leg in different spotting situations during different phases of the pirouettes endehors (Table 2). It has been demonstrated that the magnitude of the forces is connected with the aesthetic of the turn and with the number of turns for a pirouette endehors [4]. The SL (supporting leg vertical force at start) did not show any significant difference in the different spotting heights for any dancer. On the other hand the GL (gesture leg vertical force at start time) showed a significant difference between the normal spotting focus and the bottom spotting focus and also between the bottom spotting focus and the high spotting focus (Table 2).

Dancers in our study were all very experienced so we did not find any significant differences in bending time on the pirouettes with different spotting. ST is usually very close to BT because when dancers are at the maximum bending of their knees they have already shifted their body weight towards foot's toes, and "turning with double-leg support phase" has almost ended. SPT is the peak of the supporting leg and happens only a few ms later than the start time. In both cases pirouettes with bottom spotting height showed a slightly longer time at start and at peak of the supporting leg. In the HTL we found a difference $(\mathrm{p}<0.01)$ between trials with different spotting heights. When a single revolution of a pirouette endehors is well executed, dancers land softly with their gesture leg and maintain the relevé position in a motionless. In pirouettes with normal spotting skilled dancers take more time to land with their toes and their heel (Table 3). 
Table 2. Vertical forces of Support and Gesture Legs (Means and SD) at the start and at the end of the pirouette, performed with different visual spotting heights. Same symbols indicate statistical significant differences $\left(F_{3,10} ; p<0.05\right)$

\begin{tabular}{|c|c|c|}
\hline Leg and Phases & Spotting & Vertical Force (N) \\
\hline \multirow[t]{4}{*}{ Support at the Start } & Bottom & $469 \pm 39$ \\
\hline & High & $460 \pm 55$ \\
\hline & Low & $463 \pm 40$ \\
\hline & Normal & $468 \pm 52$ \\
\hline \multirow[t]{4}{*}{ Gesture at the Start } & Bottom*\# & $442 \pm 58$ \\
\hline & High $^{* \#}$ & $475 \pm 43$ \\
\hline & Low * & $457 \pm 48$ \\
\hline & Normal* & $447 \pm 50$ \\
\hline \multirow[t]{4}{*}{ Support at the End } & Bottom $^{\wedge}$ & $691 \pm 149$ \\
\hline & $\mathrm{High}^{\S}$ & $654 \pm 120$ \\
\hline & Low $^{\circ}$ & $630 \pm 94$ \\
\hline & Normal $^{\wedge \S^{\circ}}$ & $653 \pm 142$ \\
\hline \multirow[t]{4}{*}{ Gesture at the End } & Bottom & $446 \pm 92$ \\
\hline & High" & $416 \pm 80$ \\
\hline & Low" & $437 \pm 62$ \\
\hline & Normal & $450 \pm 81$ \\
\hline
\end{tabular}

Table 3. Times of the phases during pirouettes performed with different visual spotting heights (Means and SD). Same symbols indicate statistical significant differences between the spotting heights $\left(F_{3,10} ; p<0.05\right)$

\begin{tabular}{llc}
\hline Events & Spotting & Mean Time (ms) \\
\hline Start time (ST) & Bottom & $1290 \pm 470$ \\
& High & $1280 \pm 390$ \\
& Low & $1300 \pm 370$ \\
& Normal & $1280 \pm 350$ \\
\hline Support Leg Peak Time (SPT) & Bottom & $2110 \pm 440$ \\
& High & $2120 \pm 380$ \\
& Low & $2170 \pm 390$ \\
& Normal & $2160 \pm 340$ \\
\hline Toe land time (TLT) & Bottom & $2330 \pm 430$ \\
& High & $2330 \pm 320$ \\
& Low & $2330 \pm 360$ \\
& Normal & $2370 \pm 240$ \\
\hline
\end{tabular}


Table 3. Continuation

\begin{tabular}{llc}
\hline Events & Spotting & Mean Time (ms) \\
\hline Heel land time (HLT) & Bottom & $2590 \pm 440$ \\
& High & $2540 \pm 300$ \\
& Low & $2510 \pm 290$ \\
& Normal & $2630 \pm 340$ \\
\hline Recovery time (RT) & Bottom $^{\circ}$ & $3550 \pm 310$ \\
& High $^{5^{\circ}}$ & $3440 \pm 390$ \\
& Low $^{\varsigma^{\circ}}$ & $2510 \pm 290$ \\
& Normal $^{\circ}$ & $3460 \pm 370$ \\
\hline
\end{tabular}

The foot displacement during the pirouette with endehors movement was measured as the distance between the starting position and the ending position of the toe marker on the supporting leg on the medio-lateral (x) and anterior-posterior $(y)$ axis of the force platform. Significant differences were observed in the distance among " $\mathrm{x}$ " axis between pirouette with the normal preferred spotting and the one with a bottom, high and low spotting. The supporting leg after the turn tends to return in a significant different position among the horizontal axis. During the pirouette turn the distance between the starting and ending position is very small compared to the other conditions, because dancer has more experience and is good in performing with this spotting height. Any significant difference was observed along the " $y$ " axis: the bottom condition seems to be the worst for dancers. The dancers usually do not usea low spotting position and so their equilibrium was unstable when they performed in that condition (Table 4).

Table 4. Foot displacements (Means and SD) during pirouettes performed with different visual spotting heights. Same symbols indicate statistical significant differences between the spotting heights $\left(F_{3,10} ; p<0.05\right)$

\begin{tabular}{lcc}
\hline Spotting conditions & X displacement $(\mathrm{mm})$ & Y displacement $(\mathrm{mm})$ \\
\hline Bottom & $58 \pm 28^{*}$ & $32 \pm 31$ \\
\hline High & $54 \pm 11^{*}$ & $24 \pm 8$ \\
\hline Low & $62 \pm 7^{*}$ & $28 \pm 28$ \\
\hline Normal & $38 \pm 7^{*}$ & $19 \pm 4$ \\
\hline
\end{tabular}




\section{DISCUSSION}

The present findings support the hypothesis that the individual's focus of attention plays a fundamental role in how ballet turns are performed. The accuracy and quality of the pirouettes depend to a great extent the visual location on which the dancer focuses in their attention while executing the skill. The preferred spotting position for the dancers in the present study was $55 \%$ of their visual axis height and their comfortable 4 th ballet position corresponded to $28 \%$ of their leg length (Table 1). This finding is different from what was found in previous studies [13]. Probably this is due to the different body size of our dancers, quite different from elite dancers as analysed by Sugano and Laws [13]. The present study found that experienced dancers exert a greater vertical force on their gesture foot when they perform a pirouette with a high spotting position in comparison with a low spotting condition. This implies that unconscious adjustment of forces distribution to maintaining postural stability occurs. Furthermore, dancers using their preferred spotting height could maintain their retire position at the end of the pirouette for a longer time and have a faster recovery time than when using a bottom or a high spotting position.

Dancers achieved a better pirouette, with less supporting foot displacement, when they used their preferred spotting position. There is a greater distance between the starting and ending position of the toe marker on the supporting leg when the lowest spotting position was used. Spotting an external point helps dancers during a pirouette because it requires to snap their eyes around, faster than the rest of their body, to face front again, letting them focuses on one single spot. Without spotting aesthetic ballet pirouettes could not exist. Differences between focus conditions affect dancer's performance and dancers have to deal with the condition that they cannot utilize the same spotting in every ballet turn [1]. Differences in the characteristic of the room, of the stage, of the lights, of the choreographer or director's needs oblige dancers to shift their height of spotting in almost every ballet turn. Thus, it is important for dancers to practice force adjustments for different spotting locations so that they will be prepared to change their spotting location when required to do so. Significant differences happened in force were found with the greatest changes because these changes produce difficulties in achieving balance and maintaining of postural stability, and foot placements and forces must be relearned. By contrast, there are no significant consequences in slightly different spotting height conditions. 


\section{REFERENCES}

1. Denardi R, Corrêa U. (2013) Effects of instructional focus on learning a classical ballet movement, the pirouette. J Dance Med Sci, 17: 18-23

2. Farrar-Baker A, Wilmerding V. (2006) Prevalence of lateral bias in the teaching of beginning and advanced ballet. J Dance Med Sci, 10: 81-84

3. Kimmerle M. (2010) Lateral bias, functional asymmetry, dance training and injuries. J Dance Med Sci, 14: 58-66

4. Laws K. (1978) Momentum transfer in dance movement. Med Probl Perform Artists, 13: 136-145

5. Laws K. (2002) Physics and the Art of Dance Understanding Movement. Oxford: Oxford University Press

6. Lin CW, Su F, Chen S. (2014) Differences of ballet turns (pirouette) performance between experienced and novice ballet dancers. Res Q Exerc Sport, 85: $330-340$

7. Lin CW, Su F, Wu H \& Lin C. (2013) Effects of leg dominance on performance of ballet turns (pirouettes) by experienced and novice dancers. J Sports Sci, 13: $1781-1788$

8. McMillan MH. (1972) A Cinematographic Analysis of Characteristic Likenesses and Differences between Skilled, Semi-Skilled and Non-Skilled Performances of Pirouettes. Denton: Texas Women's University

9. Meglin J, Woollacott M. (1992) The neural choreography underlying a pirouette-arabesque. Kinesiol Med Dance, 14: 95-105

10. Newell KM. (1991) Motor skill acquisition. Annu Rev Psychol, 42: 213-237

11. Nigmatullina Y, Hellyer P, Nachev P, Sharp D, Seemungal B. (2015) The neuroanatomical correlates of training-related perceptuo-reflex uncoupling in dancers. Cereb Cortex, 25: 554-562

12. Payne VG, Isaacs LD. (2005) Human Motor Development: A Lifespan Approach, 6th ed. Boston: McGraw-Hill

13. Sugano MFA, Laws K. (2002) Physical analysis as a foundation for pirouette training. Med Probl Perform Artists, 17: 29-32

14. Wulf G. (2007) Attentional focus and motor learning: a review of 10 years of research. E-Journal Bewegung und Training, 4-14 http://www.sportwissenschaft.de/fileadmin/pdf/BuT/hossner_wulf.pdf

\section{Correspondence to:}

Antonio Cicchella PhD

Department for Quality of Life Studies

University of Bologna

Via Del Pilastro 8, 40127 Bologna

Italy

E-mail: antonio.cicchella@unibo.it

Tel. +39-339-3355886 